\title{
Double parton distributions for a positroniumlike bound state using light-front wave functions
}

\author{
Chandan Mondal@,$^{1, *}$ Asmita Mukherjee, ${ }^{2, \dagger}$ and Sreeraj Nair $\oplus^{1, \$}$ \\ ${ }^{1}$ Institute of Modern Physics, Chinese Academy of Sciences, Lanzhou 730000, China \\ ${ }^{2}$ Department of Physics, Indian Institute of Technology Bombay, Powai, Mumbai 400076, India
}

(Received 9 July 2019; revised manuscript received 25 September 2019; published 1 November 2019)

We investigate the double parton distributions (DPDs) for a positroniumlike bound state using light-front QED. We incorporate the higher Fock three-particle component of the state, which includes a photon. We obtain the overlap representation of the DPDs in terms of the three-particle light-front wave functions. Our calculation explores the correlations between the momentum fractions of the particles probed and the transverse distance between them, without any assumption of factorization between them. We also investigate the behavior of the DPDs near the kinematical boundary when the sum of the momentum fractions is close to 1 .

DOI: $10.1103 /$ PhysRevD.100.094002

\section{INTRODUCTION}

As the flux of partons increases in high-energy hadronic collision experiments, the probability of having more than one independent hard scattering interaction also increases, and a proper description of final states in hadronic collisions requires the inclusion of multiple partonic interactions (MPIs). The MPIs in hadronic collision were predicted long ago [1-5]. The most probable and the simplest of these MPIs are the double parton scattering (DPS) events. In DPS, two partons from each hadron participate in separate hard interactions. In such a process, a large momentum transfer is involved in both scattering. The first experimental evidence of DPS was found at CERN-ISR [6] in p-p collision. DPS events are indeed relevant at the LHC and Tevatron because of the high density of partons [7-9]. The ATLAS Collaboration reported their first results on DPS a while ago [10], and DPS also contributes to the Higgs production background in several channels at the LHC.

DPS can be factorized in terms of the hard interactions, which are calculable in perturbation theory and the double parton distribution (DPDs) functions. The DPDs depend on two-body quantities encoding the nonperturbative dynamics of the partons. Factorization of DPS usually assumes the

\footnotetext{
*mondal@impcas.ac.cn

†asmita@phy.iitb.ac.in

*sreeraj@impcas.ac.cn
}

Published by the American Physical Society under the terms of the Creative Commons Attribution 4.0 International license. Further distribution of this work must maintain attribution to the author(s) and the published article's title, journal citation, and DOI. Funded by SCOAP ${ }^{3}$. simplest case wherein there are no correlations between the two partons [11-14]. The DPDs are interpreted as the number densities of a parton pair at a given transverse distance $\mathbf{y}^{\perp}$ and carrying longitudinal momentum fractions $\left(x_{1}, x_{2}\right)$ of the composite system [2,13,15]. Since the DPDs depend on the partonic interdistance [15], they contain information on the hadronic structure that complements the tomographical information encoded by the one-body distributions such as generalized parton distributions (GPDs) [16] and transverse momentum-dependent distributions [17]. Therefore, DPDs represent a novel tool to access the three-dimensional hadron structure [18]. Despite the wealth of information provided by the DPDs, the present experimental knowledge is mainly accessible through the DPS cross section, which has been accumulated into the effective cross section, $\sigma_{\text {eff }}$. For the recent results, we refer to Refs. [19-24].

DPDs, being nonperturbative in nature, are always very difficult to evaluate from QCD first principles, and there have been numerous attempts to gain insight into them by studying QCD-inspired models. Model calculations of DPDs are important and interesting to understand the properties as well as for predictions of experimental observables. Several phenomenological models such as the bag model [25], constituent quark model [26-29], generalized valon model [30], soft-wall anti-de Sitter (AdS)/QCD model [31], dressed quark model [32], etc., have been used to obtain the basic information on DPDs and to gauge the phenomenological impact of transverse and longitudinal correlations, along with spin correlations [27,33-36]. The transverse structure of the proton from the DPDs and the effective cross section has been investigated in Refs. [15,37,38]. Recently, the matching of both the position and momentum space DPDs onto ordinary parton 
distribution functions at the next-to-leading order in perturbation theory has been reported in Ref. [39], while the authors have also discussed the sum rules for DPDs $[40,41]$. The quantities related to DPDs, and encoding double parton correlations, have been evaluated for the pion in lattice QCD [42].

As very little is known so far about the DPDs $F\left(x_{1}, x_{2}, \mathbf{y}^{\perp}\right)$, there are several approaches to parametrize or model them. A common approach is based on a factorized ansatz, which assumes that the $\mathbf{y}^{\perp}$ dependence is factored out from the dependence on $x_{1}$ and $x_{2}$, which are the momentum fractions of the partons probed. In addition, it is sometimes also assumed that $x_{1}$ and $x_{2}$ dependences are factored out, in terms of single parton distributions (PDFs) and neglecting any correlations between them [25]. Reference [43] used an approach based on Ref. [44] in which the DPDs were written as a convolution of two impact parameter-dependent PDFs, which were obtained from GPDs. A Gaussian form of the impact parameterdependent PDFs were used. However, it was concluded that the factorized ansatz fails in the valence region, and the authors also observed that a Gaussian dependence on $\mathbf{y}^{\perp}$ is rather arbitrary. It is thus relevant to investigate the DPDs without such assumptions. The model calculations can be thought of as a parametrization of the DPDs at a low momentum scale, and one then evolves them to a higher scale of the experiments, using the evolution equation; such evolution equations have been obtained by now and discussed in detail [45]. Another interesting aspect of model calculation of the DPDs is the behavior near the kinematical bound $x_{1}+x_{2}=1$. The DPDs should vanish in the unphysical region $x_{1}+x_{2}>1$. In some early model calculations, this support property was violated, due to nonconservation of momentum of the constituents. In later calculations, a phenomenological factor is included to improve the behavior in this kinematical limit [46].

A widely used method to calculate the DPDs is by expressing them in terms of overlaps of light-front wave functions (LFWFs). In the light-front (LF) formalism, the proton state is expanded in Fock space in terms of multiparton LFWFs. The LFWFs satisfy the bound-state equation in LF QCD. One then truncates the Fock space to a few-particle sector; such a truncation is boost invariant in the LF framework. As it is very difficult to solve the LF bound-state equation, in particular to obtain the wave functions of the higher Fock sector, most model calculations are restricted to using the three-quark valence LFWF for the proton. In a previous work [32], to calculate the quark-gluon DPDs, a different approach was used; namely, instead of a proton state, a relativistic spin- $1 / 2$ composite state of a quark dressed with a gluon was used. The LFWFs of the two-particle state were calculated in perturbation theory. This may be thought of as a field theorybased perturbative model, to investigate the quark-gluon correlations in the DPDs. However, the kinematics of a two-particle system are rather constrained. In this work, we use the overlap approach in terms of LFWFs for a twoparticle bound state like a positronium in QED, in the weak coupling limit. We include the effect of the three-particle $e^{+} e^{-} \gamma$ component of the LFWF. As solving the LF boundstate equation is rather difficult in QED as well, we use a simpler but nevertheless interesting approach followed earlier in Ref. [47] to calculate the twist-4 structure function of positronium and verifying a sum rule. We use an analytic form of the two-particle LFWF in the weak coupling limit. The three-particle LFWF is then expressed in terms of the two-particle LFWF using the LF QED Hamiltonian. This calculation illustrates the formalism, which can also be applied to a QCD mesonic system; in fact, in the weak coupling limit, the LFWFs are expected to mimic those of a meson. Our approach allows us to calculate them without any assumption on factorization of the $x_{1}, x_{2}$, and $\mathbf{y}^{\perp}$ dependence, and we can investigate the interplay between these variables in full form. Thus, our calculation may be thought of as an exploratory analysis on the explicit $x_{1}, x_{2}$, and $\mathbf{y}^{\perp}$ dependence of the DPDs in a three-particle system. We also discuss the behavior of the DPD in the limit $x_{1}+x_{2} \rightarrow 1$.

The paper is organized as follows. In Sec. II, we discuss the DPDs for the electron-positron pair and their overlap representation in the LF dressed positronium model. We present the numerical results in Sec. III. Conclusions are given in Sec. IV.

\section{DOUBLE PARTON DISTRIBUTIONS}

The DPDs for unpolarized quarks can be defined as $[13,14]$

$$
\begin{aligned}
F_{a_{1} a_{2}}\left(x_{1}, x_{2}, \mathbf{y}^{\perp}\right)= & 2 p^{+} \int \frac{d z_{1}^{-}}{2 \pi} \frac{d z_{2}^{-}}{2 \pi} d y^{-} e^{i\left(x_{1} z_{1}^{-}+x_{2} z_{2}^{-}\right) p^{+}} \\
& \times\left\langle p\left|\mathcal{O}_{a_{2}}\left(0, z_{2}\right) \mathcal{O}_{a_{1}}\left(y, z_{1}\right)\right| p\right\rangle,
\end{aligned}
$$

where $|p\rangle$ is the target system with momentum $p \cdot x_{1}$ and $x_{2}$ are momentum fractions of the partons, and $\mathbf{y}^{\perp}$ is the relative transverse distance between them.

The fermionic operators are given by [13] (see the Appendix)

$\mathcal{O}_{a_{i}}\left(y, z_{i}\right)=\left.\bar{\psi}_{i}\left(y-\frac{z_{i}}{2}\right) \Gamma_{a_{i}} \psi_{i}\left(y+\frac{z_{i}}{2}\right)\right|_{z_{i}^{+}=y_{i}^{+}=0, \mathbf{z}_{\mathbf{i}}^{\perp}=0}$,

where $\Gamma_{a_{i}}$ are various Dirac $\gamma$ matrices projecting onto the corresponding polarization states given by

$\Gamma_{q}=\frac{1}{2} \gamma^{+}, \quad \Gamma_{\Delta q}=\frac{1}{2} \gamma^{+} \gamma_{5}, \quad \Gamma_{\delta q}^{j}=\frac{1}{2} i \sigma^{j+} \gamma_{5}$, 
for the unpolarized fermion $(q)$, longitudinally polarized fermion $(\Delta q)$, and the transversely polarized fermion $(\delta q)$, respectively. We choose the light-cone gauge, and the gauge link in the operator structure is set to unity.

\section{A. Overlap representation for the DPD}

As discussed in the Introduction, we consider our target state to be a positroniumlike bound state in LF QED. We use the two-component form of the LF QED along the lines of Refs. [48,49]. In this section, we present a calculation of the unpolarized fermion DPDs for such a state. This means $\Gamma_{q}=\frac{1}{2} \gamma^{+}$in Eq. (2). The state can be expanded in Fock space in terms of LFWFs as

$$
\begin{aligned}
|P\rangle= & \sum_{\sigma_{1}, \sigma_{2}} \int \frac{d p_{1}^{+} d^{2} \mathbf{p}_{1}^{\perp}}{\sqrt{2(2 \pi)^{3} p_{1}^{+}}} \int \frac{d p_{2}^{+} d^{2} \mathbf{p}_{2}^{\perp}}{\sqrt{2(2 \pi)^{3} p_{2}^{+}}} \phi^{2}\left(P \mid p_{1}, \sigma_{1} ; p_{2}, \sigma_{2}\right) \sqrt{2\left((2 \pi)^{3} P^{+}\right.} \delta^{3}\left(P-p_{1}-p_{2}\right) b^{\dagger}\left(p_{1}, \sigma_{1}\right) d^{\dagger}\left(p_{2}, \sigma_{2}\right)|0\rangle \\
& +\sum_{\sigma_{1}, \sigma_{2}, \lambda} \int \frac{d p_{1}^{+} d^{2} \mathbf{p}_{1}^{\perp}}{\sqrt{2(2 \pi)^{3} p_{1}^{+}}} \int \frac{d p_{2}^{+} d^{2} \mathbf{p}_{2}^{\perp}}{\sqrt{2(2 \pi)^{3} p_{2}^{+}}} \int \frac{d p_{3}^{+} d^{2} \mathbf{p}_{\mathbf{3}}^{\perp}}{\sqrt{2(2 \pi)^{3} p_{3}^{+}}} \phi^{3}\left(P \mid p_{1}, \sigma_{1} ; p_{2}, \sigma_{2} ; p_{3}, \lambda\right) \sqrt{2(2 \pi)^{3} P^{+}} \delta^{3}\left(P-p_{1}-p_{2}-p_{3}\right) \\
& \times b^{\dagger}\left(p_{1}, \sigma_{1}\right) d^{\dagger}\left(p_{2}, \sigma_{2}\right) a^{\dagger}\left(p_{3}, \lambda\right)|0\rangle,
\end{aligned}
$$

where the first term corresponds the two-particle Fock sector, $\left|e^{+} e^{-}\right\rangle$, with the two particle LFWF $\phi^{2}$ and the second term is the three-particle Fock component, $\left|e^{+} e^{-} \gamma\right\rangle$, wherein $\phi^{3}$ is the three-particle LFWF. $\sigma_{1}, \sigma_{2}$, and $\lambda$ are the helicities of the electron, positron, and photon, respectively. The LFWF are written in terms of the Jacobi momenta $\left(x_{i}, \mathbf{q}_{\mathbf{i}}^{\perp}\right)$ defined as

$$
p_{i}^{+}=x_{i} p^{+}, \quad \mathbf{p}_{\mathbf{i}}^{\perp}=\mathbf{q}_{\mathbf{i}}^{\perp}+x_{i} \mathbf{p}^{\perp},
$$

where $\sum_{i} x_{i}=1$ and $\sum_{i} \mathbf{q}_{\mathbf{i}}^{\perp}=0$. The contribution coming from the three-particle sector of the Fock space can then be written in terms of the overlap of LFWFs,

$$
\begin{aligned}
F_{e^{-} e^{+}}\left(x_{1}, x_{2}, \mathbf{y}^{\perp}\right)= & \frac{\left(p^{+}\right)^{2}}{2 \pi^{2}} \sum_{\sigma_{1}, \sigma_{1}^{\prime}, \sigma_{2}, \sigma_{2}^{\prime}, \lambda} \int d^{2} \mathbf{k}_{1}^{\perp} d^{2} \mathbf{k}_{2}^{\perp} d^{2} \mathbf{k}_{1}^{\prime \perp} \phi_{\sigma_{1},-\sigma_{2}^{\prime}, \lambda}^{3 *}\left(p, k_{1}, k_{1}^{\prime}, p-k_{1}-k_{1}^{\prime}\right) \\
& \times \phi_{\sigma_{1}^{\prime},-\sigma_{2}, \lambda}^{3}\left(p, k_{1}+k_{1}^{\prime}-k_{2}, p-k_{1}-k_{1}^{\prime}\right) e^{i\left(\mathbf{k}_{1}^{\perp}-\mathbf{k}_{1}^{\prime \perp}\right) \cdot \mathbf{y}^{\perp}},
\end{aligned}
$$

with $p^{+} \phi_{\sigma_{1} \sigma_{2} \lambda}^{3}\left(k_{i}^{+}, \mathbf{k}_{\mathbf{i}}^{\perp}\right)=\psi_{\sigma_{1} \sigma_{2} \lambda}^{3}\left(x_{i}, \mathbf{q}_{\mathbf{i}}^{\perp}\right)$. We can rewrite Eq. (6) as

$$
\begin{aligned}
F_{e^{-} e^{+}}\left(x_{1}, x_{2}, \mathbf{y}^{\perp}\right)= & \frac{1}{2 \pi^{2}} \sum_{\sigma_{1}, \sigma_{1}^{\prime}, \sigma_{2}, \sigma_{2}^{\prime}, \lambda} \int d^{2} \mathbf{k}_{\mathbf{1}}^{\perp} d^{2} \mathbf{k}_{\mathbf{2}}^{\perp} d^{2} \mathbf{k}_{\mathbf{1}}^{\perp} \psi_{\sigma_{1},-\sigma_{2}^{\prime}, \lambda}^{3 *}\left(x_{1}, \mathbf{k}_{\mathbf{1}}^{\perp} ; x_{2}, \mathbf{k}_{\mathbf{1}}^{\prime \perp}+\mathbf{k}_{\mathbf{2}}^{\perp}-\mathbf{k}_{\mathbf{1}}^{\perp} ; 1-x_{1}-x_{2}, \mathbf{k}_{\mathbf{3}}^{\perp}\right) \\
& \times \psi_{\sigma_{1}^{\prime},-\sigma_{2}, \lambda}^{3}\left(x_{1}, \mathbf{k}_{\mathbf{1}}^{\perp \perp} ; x_{2}, \mathbf{k}_{\mathbf{2}}^{\perp} ; 1-x_{1}-x_{2}, \mathbf{k}_{\mathbf{3}}^{\perp}\right) e^{i\left(\mathbf{k}_{1}^{\perp}-\mathbf{k}_{1}^{\prime \perp}\right) \mathbf{y}^{\perp}},
\end{aligned}
$$

where $\mathbf{k}_{\mathbf{3}}^{\perp}=\mathbf{p}^{\perp}-\mathbf{k}_{\mathbf{1}}^{\prime \perp}-\mathbf{k}_{\mathbf{2}}^{\perp}$, we consider the frame in which $\mathbf{p}^{\perp}=0$. The amplitudes or LFWFs $\psi^{2}$ and $\psi^{3}$ are boost invariant and are functions of the Jacobi momenta. These can be written as [49,50]

$$
\psi_{\sigma_{1}, \sigma_{2}, \lambda_{3}}^{3}\left(x_{1}, k_{1} ; x_{2}, k_{2} ; 1-x_{1}-x_{2}, k_{3}\right)=\mathcal{M}_{1}+\mathcal{M}_{2},
$$

where the amplitudes are given by [47]

$$
\begin{aligned}
& \mathcal{M}_{1}=\frac{1}{E}(-) \frac{e}{\sqrt{2(2 \pi)^{3}}} \frac{1}{\sqrt{1-x_{1}-x_{2}}} V_{1} \psi_{s_{1}, \sigma_{2}}^{2}\left(1-x_{2},-\mathbf{k}_{\mathbf{2}}^{\perp} ; x_{2}, \mathbf{k}_{2}^{\perp}\right), \\
& \mathcal{M}_{2}=\frac{1}{E} \frac{e}{\sqrt{2(2 \pi)^{3}}} \frac{1}{\sqrt{1-x_{1}-x_{2}}} V_{2} \psi_{\sigma_{1}, s_{2}}^{2}\left(x_{1}, \mathbf{k}_{1}^{\perp} ; 1-x_{1},-\mathbf{k}_{1}^{\perp}\right),
\end{aligned}
$$

with the energy denominator 


$$
E\left(x_{1}, x_{2}\right)=\left[M^{2}-\frac{m^{2}+\left(\mathbf{k}_{\mathbf{1}}^{\perp}\right)^{2}}{x_{1}}-\frac{m^{2}+\left(\mathbf{k}_{\mathbf{2}}^{\perp}\right)^{2}}{x_{2}}-\frac{\left(\mathbf{k}_{\mathbf{3}}^{\perp}\right)^{2}}{1-x_{1}-x_{2}}\right]
$$

and the vertices

$$
\begin{aligned}
& V_{1}\left(x_{1}, \mathbf{k}_{\mathbf{1}}^{\perp} ; x_{2}, \mathbf{k}_{\mathbf{2}}^{\perp}\right)=\chi_{\sigma_{1}}^{\dagger} \sum_{s_{1}}\left[\frac{2 \mathbf{k}_{\mathbf{3}}^{\perp}}{1-x_{1}-x_{2}}-\frac{\left(\sigma^{\perp} \cdot \mathbf{k}_{\mathbf{1}}^{\perp}-i m\right)}{x} \sigma^{\perp}+\sigma^{\perp} \frac{\left(\sigma^{\perp} \cdot \mathbf{k}_{\mathbf{2}}^{\perp}-i m\right)}{1-x_{2}}\right] \chi_{s_{1}} \cdot\left(\epsilon_{\lambda_{1}}^{\perp}\right)^{*}, \\
& V_{2}\left(x_{1}, \mathbf{k}_{\mathbf{1}}^{\perp} ; x_{2}, \mathbf{k}_{\mathbf{2}}^{\perp}\right)=\chi_{-\sigma_{2}}^{\dagger} \sum_{s_{2}}\left[\frac{2 \mathbf{k}_{\mathbf{3}}^{\perp}}{1-x_{1}-x_{2}}-\sigma^{\perp} \frac{\left(\sigma^{\perp} \cdot \mathbf{k}_{\mathbf{2}}^{\perp}-i m\right)}{x_{2}}+\frac{\left(\sigma^{\perp} \cdot \mathbf{k}_{\mathbf{1}}^{\perp}-i m\right)}{1-x_{1}} \sigma^{\perp}\right] \chi_{-s_{2}} \cdot\left(\epsilon_{\lambda_{1}}^{\perp}\right)^{*} .
\end{aligned}
$$

The above expressions are obtained using the LF Hamiltonian for QED along a similar line as in LF QCD [49]. Following Eqs. (8)-(10), we can rewrite Eq. (7) in terms of $\psi^{2}$ as

$$
F_{e^{-} e^{+}}\left(x_{1}, x_{2}, \mathbf{y}^{\perp}\right)=\frac{e^{2}}{(2 \pi)^{5}} \frac{1}{\left[E\left(x_{1}, x_{2}\right)\right]^{2}} \frac{1}{1-x_{1}-x_{2}} \sum_{\sigma_{1}, \sigma_{1}^{\prime}, \sigma_{2}, \sigma_{2}^{\prime}, \lambda} \int d^{2} \mathbf{k}_{1}^{\perp} d^{2} \mathbf{k}_{2}^{\perp} d^{2} \mathbf{k}_{\mathbf{1}}^{\perp \perp} \times\left[\mathcal{P}_{11}+\mathcal{P}_{12}+\mathcal{P}_{21}+\mathcal{P}_{22}\right] e^{i\left(\mathbf{k}_{1}^{\perp}-\mathbf{k}_{1}^{\prime \perp}\right) \cdot \mathbf{y}^{\perp}},
$$

where

$$
\begin{aligned}
\mathcal{P}_{11}= & {\left[V_{1}\left(x_{1}, \mathbf{k}_{\mathbf{1}}^{\perp} ; x_{2}, \mathbf{k}_{\mathbf{1}}^{\prime \perp}+\mathbf{k}_{\mathbf{2}}^{\perp}-\mathbf{k}_{\mathbf{1}}^{\perp}\right) \psi_{s_{1},-\sigma_{2}^{\prime}}^{2}\left(1-x_{2},-\mathbf{k}_{\mathbf{1}}^{\perp \perp}-\mathbf{k}_{\mathbf{2}}^{\perp}+\mathbf{k}_{\mathbf{1}}^{\perp} ; x_{2}, \mathbf{k}_{\mathbf{1}}^{\prime \perp}+\mathbf{k}_{\mathbf{2}}^{\perp}-\mathbf{k}_{\mathbf{1}}^{\perp}\right)\right]^{\dagger} } \\
& \times\left[V_{1}\left(x_{1}, \mathbf{k}_{\mathbf{1}}^{\prime \perp} ; x_{2}, \mathbf{k}_{\mathbf{2}}^{\perp}\right) \psi_{s_{1},-\sigma_{2}}^{2}\left(1-x_{2},-\mathbf{k}_{\mathbf{2}}^{\perp} ; x_{2}, \mathbf{k}_{\mathbf{2}}^{\perp}\right)\right], \\
\mathcal{P}_{22}= & {\left[V_{2}\left(x_{1}, \mathbf{k}_{\mathbf{1}}^{\perp} ; x_{2}, \mathbf{k}_{\mathbf{1}}^{\perp}+\mathbf{k}_{\mathbf{2}}^{\perp}-\mathbf{k}_{\mathbf{1}}^{\perp}\right) \psi_{\sigma_{1}, s_{2}}^{2}\left(x_{1}, \mathbf{k}_{\mathbf{1}}^{\perp} ; 1-x_{1},-\mathbf{k}_{\mathbf{1}}^{\perp}\right)\right]^{\dagger} } \\
& \times\left[V_{2}\left(x_{1}, \mathbf{k}_{\mathbf{1}}^{\perp \perp} ; x_{2}, \mathbf{k}_{\mathbf{2}}^{\perp}\right) \psi_{\sigma_{1}^{\prime}, s_{2}}^{2}\left(x_{1}, \mathbf{k}_{\mathbf{1}}^{\prime \perp} ; 1-x_{1},-\mathbf{k}_{\mathbf{1}}^{\prime \perp}\right)\right], \\
\mathcal{P}_{12}= & {\left[V_{1}\left(x_{1}, \mathbf{k}_{\mathbf{1}}^{\perp} ; x_{2}, \mathbf{k}_{\mathbf{1}}^{\perp}+\mathbf{k}_{\mathbf{2}}^{\perp}-\mathbf{k}_{\mathbf{1}}^{\perp}\right) \psi_{s_{1},-\sigma_{2}^{\prime}}^{2}\left(1-x_{2},-\mathbf{k}_{\mathbf{1}}^{\perp \perp}-\mathbf{k}_{\mathbf{2}}^{\perp}+\mathbf{k}_{\mathbf{1}}^{\perp} ; x_{2}, \mathbf{k}_{\mathbf{1}}^{\prime \perp}+\mathbf{k}_{\mathbf{2}}^{\perp}-\mathbf{k}_{\mathbf{1}}^{\perp}\right)\right]^{\dagger} } \\
& \times\left[V_{2}\left(x_{1}, \mathbf{k}_{\mathbf{1}}^{\prime \perp} ; x_{2}, \mathbf{k}_{\mathbf{2}}^{\perp}\right) \psi_{\sigma_{1}^{\prime}, s_{2}}^{2}\left(x_{1}, \mathbf{k}_{\mathbf{1}}^{\prime \perp} ; 1-x_{1},-\mathbf{k}_{\mathbf{1}}^{\prime \perp}\right)\right], \\
\mathcal{P}_{21}= & {\left[V_{2}\left(x_{1}, \mathbf{k}_{\mathbf{1}}^{\perp} ; x_{2}, \mathbf{k}_{\mathbf{1}}^{\perp}+\mathbf{k}_{\mathbf{2}}^{\perp}-\mathbf{k}_{\mathbf{1}}^{\perp}\right) \psi_{\sigma_{1}, s_{2}}^{2}\left(x_{1}, \mathbf{k}_{\mathbf{1}}^{\perp} ; 1-x_{1},-\mathbf{k}_{\mathbf{1}}^{\perp}\right)\right]^{\dagger} } \\
& \times\left[V_{1}\left(x_{1}, \mathbf{k}_{\mathbf{1}}^{\prime \perp} ; x_{2}, \mathbf{k}_{\mathbf{2}}^{\perp}\right) \psi_{s_{1},-\sigma_{2}}^{2}\left(1-x_{2},-\mathbf{k}_{\mathbf{2}}^{\perp} ; x_{2}, \mathbf{k}_{\mathbf{2}}^{\perp}\right)\right] .
\end{aligned}
$$

The above expression is evaluated using Mathematica to calculate the spinor products. The final expressions are given below:

$$
\begin{gathered}
\mathcal{P}_{11}=\frac{8\left(1+x_{1}-x_{2}\right)^{2}\left(k_{1}^{\prime y}\left(1-x_{2}\right)+k_{2}^{y} x_{1}\right)\left(\left(k_{1}^{\prime y}+k_{2}^{y}\right) x_{1}-k_{1}^{y}\left(x_{1}+x_{2}-1\right)\right)}{x_{1}^{2}\left(x_{2}-1\right)^{2}\left(x_{1}+x_{2}-1\right)^{2}} \times \psi^{2}\left(x_{2}, \mathbf{k}_{1}^{\perp}-\mathbf{k}_{1}^{\prime \perp}-\mathbf{k}_{2}^{\perp}\right) \psi^{2}\left(x_{2}, \mathbf{k}_{\mathbf{2}}^{\perp}\right), \\
\mathcal{P}_{22}=\frac{-8\left(1-x_{1}+x_{2}\right)^{2}\left(k_{2}^{y}\left(x_{1}-1\right)+k_{1}^{\prime y} x_{2}\right)\left(k_{1}^{y}\left(x_{1}+x_{2}-1\right)-\left(k_{1}^{\prime y}+k_{2}^{y}\right)\left(x_{1}-1\right)\right)}{x_{2}^{2}\left(x_{1}-1\right)^{2}\left(x_{1}+x_{2}-1\right)^{2}} \times \psi^{2}\left(x_{1}, \mathbf{k}_{1}^{\perp}\right) \psi^{2}\left(x_{1}, \mathbf{k}_{1}^{\prime \perp}\right), \\
\mathcal{P}_{12}=\frac{8\left(\left(x_{1}-x_{2}\right)^{2}-1\right)\left(k_{2}^{y}\left(1-x_{1}\right)+k_{1}^{\prime y} x_{2}\right)\left(k_{1}^{y}\left(x_{1}+x_{2}-1\right)-\left(k_{1}^{y}+k_{2}^{y}\right) x_{1}\right)}{x_{1}\left(x_{1}-1\right) x_{2}\left(x_{2}-1\right)\left(x_{1}+x_{2}-1\right)^{2}} \times \psi^{2}\left(x_{2}, \mathbf{k}_{\mathbf{1}}^{\perp}-\mathbf{k}_{\mathbf{1}}^{\prime \perp}-\mathbf{k}_{\mathbf{2}}^{\perp}\right) \psi^{2}\left(x_{1}, \mathbf{k}_{1}^{\prime \perp}\right), \\
\mathcal{P}_{21}=\frac{8\left(\left(x_{1}-x_{2}\right)^{2}-1\right)\left(k_{1}^{\prime y}\left(1-x_{2}\right)+k_{2}^{y} x_{1}\right)\left(\left(k_{1}^{\prime y}+k_{2}^{y}\right)\left(x_{1}-1\right)-k_{1}^{y}\left(x_{1}+x_{2}-1\right)\right)}{x_{1}\left(x_{1}-1\right) x_{2}\left(x_{2}-1\right)\left(x_{1}+x_{2}-1\right)^{2}} \times \psi^{2}\left(x_{1}, \mathbf{k}_{\mathbf{1}}^{\perp}\right) \psi^{2}\left(x_{2}, \mathbf{k}_{2}^{\perp}\right) .
\end{gathered}
$$

Motivated by Refs. [47,51], we take the two-particle wave function $\psi^{2}$ in the weak coupling limit as 


$$
\psi^{2}\left(x, \mathbf{k}^{\perp}\right)=\sqrt{\frac{m}{\pi^{2}}} \frac{4\left(e_{1}\right)^{5 / 2}}{\left[\left(e_{1}\right)^{2}-m^{2}+\frac{1}{4} \frac{\left(\mathbf{k}^{\perp}\right)^{2}+m^{2}}{x(1-x)}\right]^{2}},
$$

with $m$ being the electron mass and $e_{1}=m / 2$.

\section{NUMERICAL RESULTS}

In this section, we present the numerical results for the unpolarized DPDs for the correlation between $e^{-}$and $e^{+}$. To do the numerical calculations, a cutoff $k_{\max }=20 \mathrm{MeV}$ has been introduced for the upper limit of all the integrations over $\mathbf{k}^{\perp}$. We notice that for higher values of $k_{\max }$ the results do not change. The electron (positron) mass has been taken as $m=0.50 \mathrm{MeV}$. In Fig. 1, we show the DPD for the $e^{-}$and $e^{+}$pair in the positroniumlike bound state as a function of $x_{1}$ for different values of $x_{2}$ and a fixed value of $\mathbf{y}^{\perp}=0.2 \mathrm{MeV}^{-1}$. In this figure, we present the contribution evaluated from the $\left|e^{-} e^{+} \gamma\right\rangle$ Fock sector. In our calculation, we have chosen the physical kinematical region in the three-particle sector, that is, $x_{1}+x_{2}<1$. We observe that as $x_{1}+x_{2} \rightarrow 1$ the distribution shows a sharp rise. This is expected since the analytic expression from the three-particle section has a pole at $x_{1}+x_{2}=1$.
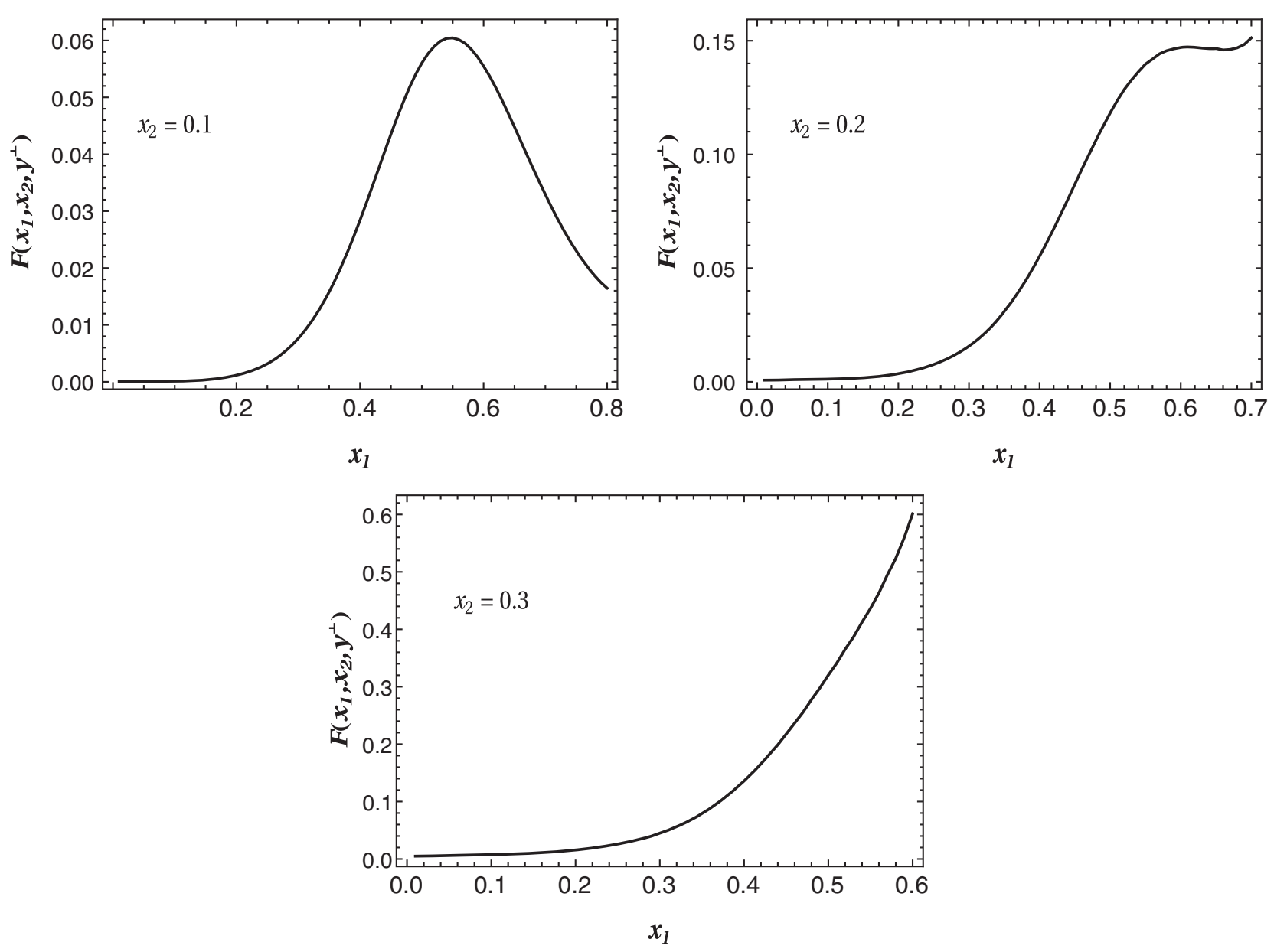

FIG. 1. Plot for $F\left(x_{1}, x_{2}, \mathbf{y}^{\perp}\right)$ vs $x_{1}$ for a fixed value of $\mathbf{y}^{\perp}=0.2 \mathrm{MeV}^{-1}$. The numbers on the vertical axis are in units of $\mathrm{MeV}^{2}$.
Actually, the two-particle sector contributes at $x_{1}+x_{2}=1$, in particular, to the normalization of the state, and it is necessary to incorporate this contribution to correctly predict the behavior of the DPDs at $x_{1}+x_{2}=1$, similar to the calculation of single parton distribution, or the structure functions [50]. In the calculation of the PDFs, the normalization contribution coming from the state cancels the pole. It is beyond the scope of the present work to investigate if such cancellation happens for the DPDs; rather, we follow a more phenomenological approach, as seen later in this section. It can also be noticed that there is a peak in the distribution near $x_{1} \approx 0.5$ for a lower value of $x_{2}$. However, the peak disappears as the magnitude of the distributions increases significantly with increasing $x_{2}$, and the distribution behaves like an ordinary parton distribution function of the bare electron in a physical electron system [52]. In Fig. 1, the peak is present only for $x_{2}=0.1$ because of the term $\left(x_{1}-1\right)^{2} x_{2}^{2}\left(x_{1}+x_{2}-1\right)^{2}$ present in the denominator of the term $\mathcal{P}_{22}$, which suppress the peak value for $x_{2}>0.1$. The DPD as a function of $x_{1}$ for four different values of $x_{2}$ together with fixed $\mathbf{y}^{\perp}=0.2 \mathrm{MeV}^{-1}$ is shown in Fig. 2 . We notice that the maximum value for the distribution is 


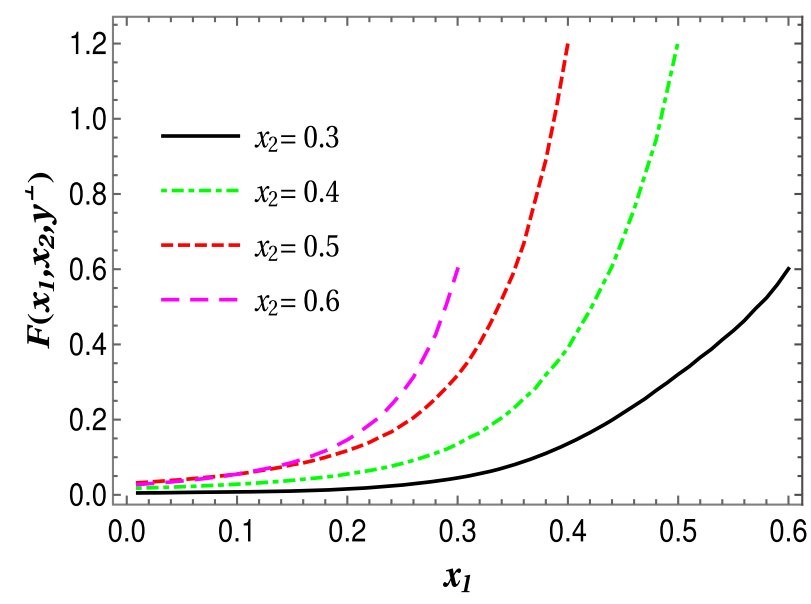

FIG. 2. Plot for $F\left(x_{1}, x_{2}, \mathbf{y}^{\perp}\right)$ as a function of $x_{1}$ for different values of $x_{2}=(0.3,0.4,0.5,0.6)$ and a fixed value of $\mathbf{y}^{\perp}=0.2 \mathrm{MeV}^{-1}$. The numbers on the vertical axis are in units of $\mathrm{MeV}^{2}$

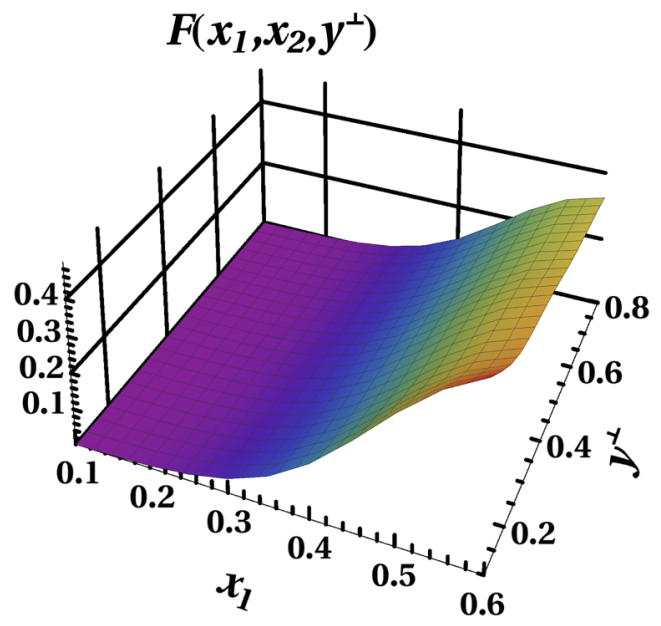

FIG. 3. 3D plot for $F\left(x_{1}, x_{2}, \mathbf{y}^{\perp}\right)$ as a function of $x_{1}$ and $\mathbf{y}^{\perp}$ for a fixed value of $x_{2}=0.3$. The numbers on the vertical axis are in units of $\mathrm{MeV}^{2}$, and $\mathbf{y}^{\perp}$ is in $\mathrm{MeV}^{-1}$.

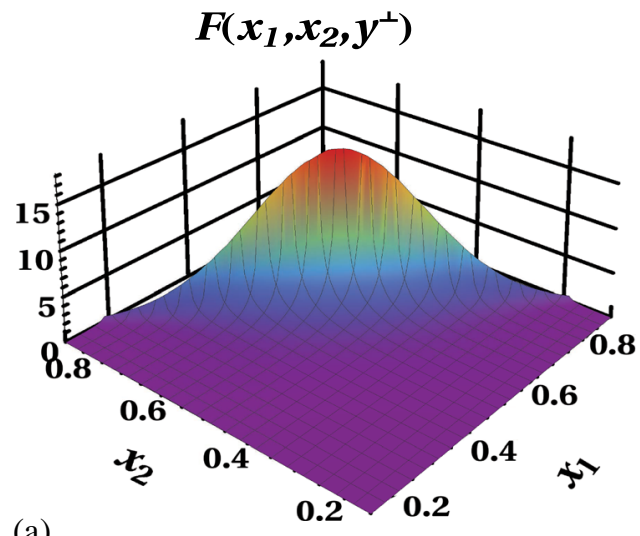

(a)

FIG. 4. (a) 3D and (b) contour plot for $F\left(x_{1}, x_{2}, \mathbf{y}^{\perp}\right)$ as a function of $x_{1}$ and $x_{2}$ for a fixed value of $\mathbf{y}^{\perp}=0.2 \mathrm{MeV}^{-1}$. The numbers for $F\left(x_{1}, x_{2}, \mathbf{y}^{\perp}\right)$ are in units of $\mathrm{MeV}^{2}$. obtained near $x_{1}=0.5$ when $x_{2}=0.5$, and the magnitude of the distribution gradually decreases on both sides of $x_{2}=0.5$. This implies that the correlation of the $e^{-} e^{+}$pair is maximum when they share the total momentum of the system equally.

Figure 3 shows the three-dimensional (3D) plot of the DPD as a function of $\mathbf{y}^{\perp}$ and $x_{1}$ for fixed values of $x_{2}=0.3$. The DPD has the maximum but finite value when the relative distance between the $e^{-}$and $e^{+}$is zero. However, it decreases gradually with an increasing value of $\mathbf{y}^{\perp}$. The magnitude of the distribution increases as the value of $x_{1}$ increases.

Figure 4(a) shows the 3D plot of the DPD as a function of $x_{1}$ and $x_{2}$ for fixed values of $\mathbf{y}^{\perp}=0.2 \mathrm{MeV}^{-1}$. The same plot with a reduced range for the magnitude of DPD is shown as a contour plot in Fig. 4(b). Both plots cover the region $x_{1}+x_{2}<1$. As observed in the twodimensional (2D) plots in Fig. 1, we observe that the magnitude of the DPD sharply increases as $x_{1}+x_{2} \rightarrow 1$. We can clearly observe the symmetry between $x_{1}$ and $x_{2}$ from these two plots.

As discussed in the Introduction, the DPDs for a three-particle system should vanish in the unphysical region $x_{1}+x_{2}>1$. In some model calculations, for example, in the bag model [25] and in the constituent quark model [26], this support property was found to be violated. To have a correct behavior near the kinematical bound $x_{1}+x_{2}=1$, it is common to introduce a factor $\theta\left(1-x_{1}-x_{2}\right)\left(1-x_{1}-x_{2}\right)^{n}$, in model calculations of the DPDs for a nucleon, where $n$ is a parameter to be determined phenomenologically [43,53]. In Ref. [45], it was found that a common factor $\left(1-x_{1}-x_{2}\right)^{n}$ multiplying all DPDs led to a violation of momentum sum rule, and the authors suggested a modification of this factor. Motivated by these results, in Fig. 5, we multiply the DPD by a factor of $\left(1-x_{1}-x_{2}\right)^{n}$, and we plot the DPD as a function of $x_{1}$ for fixed values of $x_{2}$ and $\mathbf{y}^{\perp}$. We show the result for three

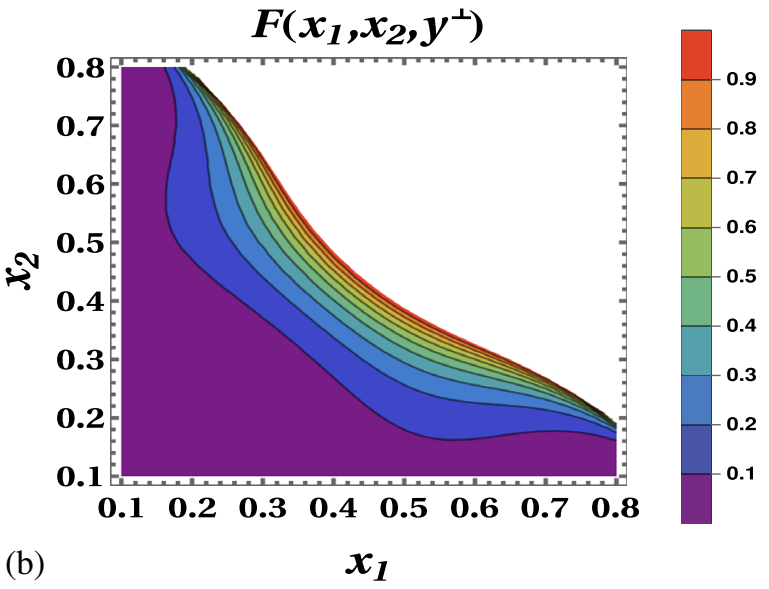

(b) 

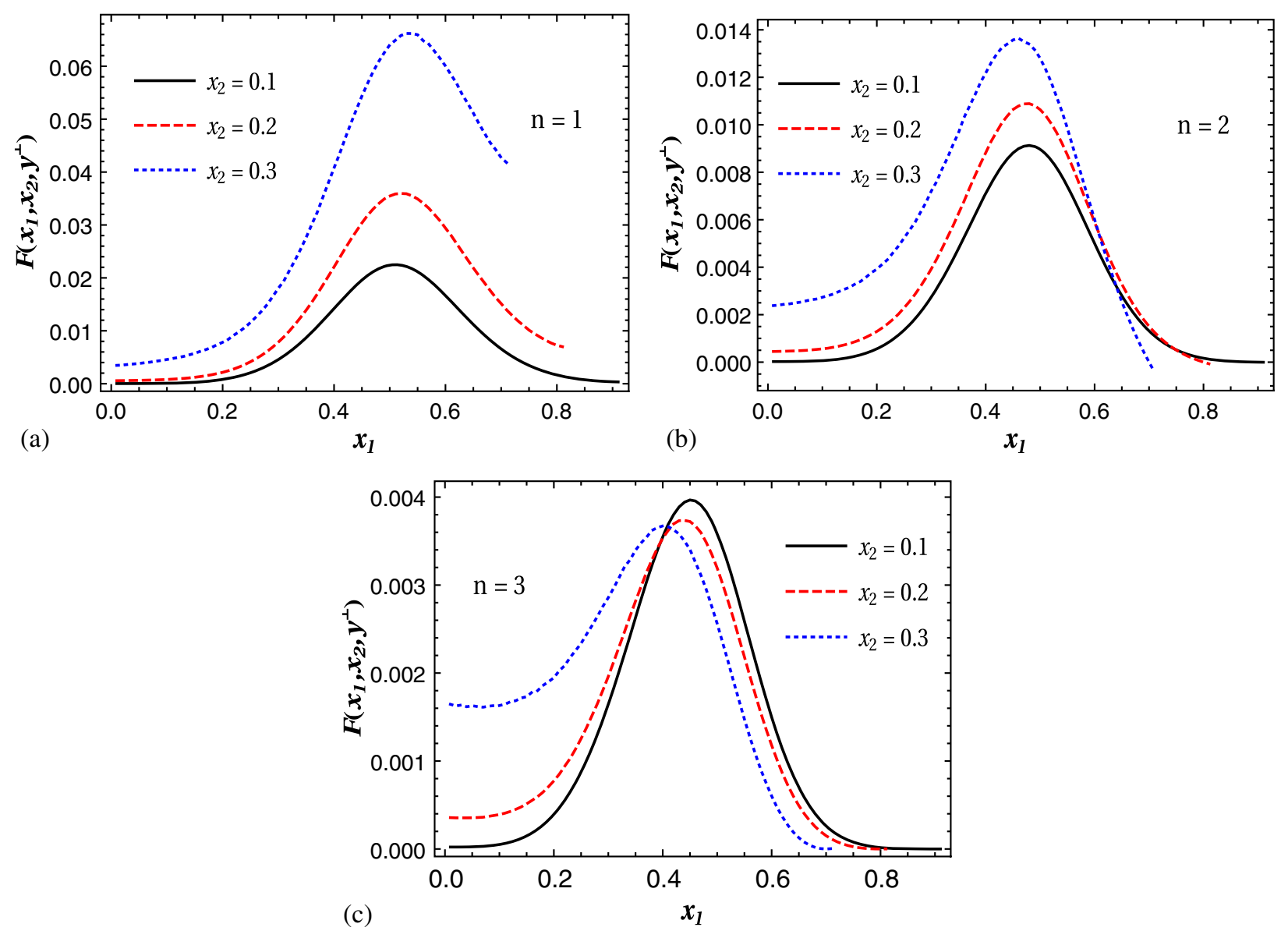

FIG. 5. Plot for $F\left(x_{1}, x_{2}, \mathbf{y}^{\perp}\right)$ vs $x_{1}$ for a fixed value of $\mathbf{y}^{\perp}=0.2 \mathrm{MeV}^{-1}$ and $x_{2}=(0.1,0.2,0.3)$. The plots $(a, b, c)$ are for three different values of the parameter $n=(1,2,3)$. The numbers on the vertical axis are in units of $\mathrm{MeV}^{2}$.

different values of $n=(1,2,3)$. The behavior near the bound $x_{1}+x_{2} \rightarrow 1$ is improved. The DPD has a peak in $x_{1}$ for a fixed value of $x_{2}$ (and vice versa); the position of the peak shifts to a lower $x_{1}$ value as $n$ increases. For a given value of $n$, the peak occurs at smaller $x_{1}$ values for larger $x_{2}$.

The phenomenological correction is used to take account of the fact that finding a parton with $x=x_{1}$ reduces the probability of finding another parton with $x=x_{2}$ if $x_{1}+x_{2}$ is close to 1 . The trimming is expected to be much smaller if $x_{1}$ were small and $x_{2}$ were large than if both $x_{1}$ and $x_{2}$ were large, even if the sum of $x_{1}$ and $x_{2}$ were the same in both cases [45]. In agreement with Ref. [45], we find that the reduction due to the phenomenological correction is large for $x_{2}=0.3$ compared to $x_{2}=0.1$ and $x_{2}=0.2$ for all three values of $n$ and the reduction is even larger for $n=3$. However, the probability should be larger when both the partons carry larger momentum. In Fig. 5, we notice that only for $n=1, x_{2}=0.3$ distribution dominates over the other distributions for $x_{2}=0.1$ and $x_{2}=0.2$. This implies that the probability of finding $\left\{e^{-}, e^{+}\right\}$with momentum fractions $\left\{x_{1}, x_{2}\right\}=\{0.3,0.7\}$ is larger than that when they carry the momentum fractions $\{0.2,0.8\}$ or
$\{0.1,0.9\}$. However, the argument is violated for $n=2$ and $n=3$. Thus, we prefer $n=1$ in our work. The phenomenological correction with $n=1$ was recommended in early papers $[3,54-56]$.

The scale evolution of the DPD is complicated by the fact that, in general, there can be two different scales corresponding to the two different hard scatterings initiated by the two partons having different virtualities. The approximation in which the two different hard scales are set to be the same is referred to as the collinear approximation. The first calculation of the generalized DGLAP equation in the collinear approximation was shown in Refs. [57,58]. The general case with two different virtualities has been reported in Ref. [59]. In our framework, the scale evolution of the DPDs could be studied using lightfront Hamiltonian perturbation theory along the lines of Ref. [50] and including the effect of the normalization of the state. This is beyond the scope of the current study. The general expectation from the evolution would be a reduction in the distribution, owing to the creation of new partonic species carrying momentum, in particular, gluon and sea quark distributions in the QCD system or photon distributions in the QED system. 


\section{CONCLUSION}

We have presented a calculation of the electron-positron unpolarized DPD for a positroniumlike bound state in LF QED. We have expressed the DPDs as overlaps of the three-particle LFWFs that include a photon. The analytic form of the LFWFs is obtained using the LF QED Hamiltonian. Our approach allows us to investigate the correlation between the momentum fractions $x_{1}$ adn $x_{2}$ and the transverse separation $\mathbf{y}^{\perp}$ of the DPDs without assuming any factorization between them and may help in improving model parametrizations of mesons DPDs. The DPDs show strong correlations between these variables. The behavior near the kinematical boundary $x_{1}+x_{2}=1$ is improved by introducing a phenomenological factor. Our calculation may act as a guide to develop models for the DPDs of mesons at the low momentum scale.

\section{ACKNOWLEDGMENTS}

The work of C. M. is supported by the China Postdoctoral Science Foundation (CPSF) under Grant No. 2017M623279 and the National Natural Science Foundation of China (NSFC) under Grant No. 11850410436. S. N. is supported by the China Postdoctoral Council under the International Postdoctoral Exchange Fellowship Program. We thank T. Kasemets and X. Zhao for helpful discussions.

\section{APPENDIX}

The operators for two unpolarized fermions correlator are given by

$$
\begin{aligned}
\mathcal{O}_{1}\left(y, z_{1}\right) & =\bar{\psi}\left(y-\frac{z_{1}}{2}\right) \gamma^{+} \psi\left(y+\frac{z_{1}}{2}\right) \\
& =2 \xi^{\dagger}\left(y-\frac{z_{1}}{2}\right) \xi\left(y+\frac{z_{1}}{2}\right) \\
\mathcal{O}_{2}\left(0, z_{2}\right) & =\bar{\psi}\left(\frac{z_{2}}{2}\right) \gamma^{+} \psi\left(-\frac{z_{2}}{2}\right) \\
& =2 \xi^{\dagger}\left(\frac{z_{2}}{2}\right) \xi\left(-\frac{z_{2}}{2}\right)
\end{aligned}
$$

with

$$
\xi(x)=\sum_{\lambda} \chi_{\lambda} \int \frac{d k^{+} d^{2} \mathbf{k}^{\perp}}{2(2 \pi)^{3} \sqrt{k^{+}}}\left(b_{\lambda}(k) e^{-i k x}+d_{-\lambda}^{\dagger}(k) e^{i k x}\right) .
$$

The fermionic operators for the DPD correlator is

$$
\begin{aligned}
\mathcal{O}_{2}\left(0, z_{2}\right) \mathcal{O}_{1}\left(y, z_{1}\right)= & \sum_{\text {spin }} \int \frac{d k_{1}^{+} d^{2} \mathbf{k}_{1}^{\perp}}{2(2 \pi)^{3} \sqrt{k_{1}^{+}}} \int \frac{d k_{1}^{\prime}+d^{2} \mathbf{k}_{1}^{\prime \perp}}{2(2 \pi)^{3} \sqrt{k_{1}^{\prime+}}} \int \frac{d k_{2}^{+} d^{2} \mathbf{k}_{2}^{\perp}}{2(2 \pi)^{3} \sqrt{k_{2}^{+}}} \int \frac{d k_{2}^{\prime+} d^{2} \mathbf{k}_{2}^{\prime \perp}}{2(2 \pi)^{3} \sqrt{k_{2}^{\prime+}}} \\
& \times\left[b_{\sigma_{2}}^{\dagger}\left(k_{2}\right) b_{\sigma_{2}^{\prime}}\left(k_{2}^{\prime}\right) b_{\sigma_{1}}^{\dagger}\left(k_{1}\right) b_{\sigma_{1}^{\prime}}\left(k_{1}^{\prime}\right) e^{i k_{1} \cdot\left(y-\frac{z_{1}}{2}\right)} e^{-i k_{1}^{\prime} \cdot\left(y+\frac{z_{1}}{2}\right)} e^{\frac{i}{2} k_{2}^{\prime} \cdot z_{2}} e^{\frac{i}{2} k_{2} \cdot z_{2}}\right. \\
& +b_{\sigma_{2}}^{\dagger}\left(k_{2}\right) b_{\sigma_{2}^{\prime}}\left(k_{2}^{\prime}{ }_{2} d_{-\sigma_{1}}\left(k_{1}\right) d_{-\sigma_{1}^{\prime}}^{\dagger}\left(k_{1}^{\prime}\right) e^{-i k_{1} \cdot\left(y-\frac{z_{1}}{2}\right)} e^{i k_{1}^{\prime} \cdot\left(y+\frac{z_{1}}{2}\right)} e^{\frac{i}{2} k_{2}^{\prime} \cdot z_{2}} e^{\frac{i}{2} k_{2} \cdot z_{2}}\right. \\
& +b_{\sigma_{2}}^{\dagger}\left(k_{2}\right) d_{-\sigma_{2}^{\prime}}^{\dagger}\left(k_{2}^{\prime}{ }_{2} d_{-\sigma_{1}}\left(k_{1}\right) b_{\sigma_{1}^{\prime}}\left(k_{1}^{\prime}\right) e^{-i k_{1} \cdot\left(y-\frac{z_{1}}{2}\right)} e^{-i k_{1}^{\prime} \cdot\left(y+\frac{z_{1}}{2}\right)} e^{-\frac{i}{2} k_{2}^{\prime} \cdot z_{2}} e^{\frac{i}{2} k_{2} \cdot z_{2}}\right. \\
& +d_{-\sigma_{2}}\left(k_{2}\right) b_{\sigma_{2}^{\prime}}\left(k_{2}^{\prime}{ }_{2}\right) b_{\sigma_{1}}^{\dagger}\left(k_{1}\right) d_{-\sigma_{1}^{\prime}}^{\dagger}\left(k_{1}^{\prime}\right) e^{i k_{1} \cdot\left(y-\frac{z_{1}}{2}\right)} e^{i k_{1}^{\prime} \cdot\left(y+\frac{z_{1}}{2}\right)} e^{\frac{i}{2} k_{2}^{\prime} \cdot z_{2}} e^{-\frac{i}{2} k_{2} \cdot z_{2}} \\
& +d_{-\sigma_{2}}\left(k_{2}\right) d_{-\sigma_{2}^{\prime}}^{\dagger}\left(k_{2}^{\prime}\right) b_{\sigma_{1}}^{\dagger}\left(k_{1}\right) b_{\sigma_{1}^{\prime}}\left(k_{1}^{\prime}\right) e^{i k_{1} \cdot\left(y-\frac{z_{1}}{2}\right)} e^{-i k_{1}^{\prime} \cdot\left(y+\frac{z_{1}}{2}\right)} e^{-\frac{i}{2} k_{2}^{\prime} \cdot z_{2}} e^{-\frac{i}{2} k_{2} \cdot z_{2}} \\
& \left.+d_{-\sigma_{2}}\left(k_{2}\right) d_{-\sigma_{2}^{\prime}}^{\dagger}\left(k_{2}^{\prime}\right) d_{-\sigma_{1}}\left(k_{1}\right) d_{-\sigma_{1}^{\prime}}^{\dagger}\left(k_{1}^{\prime}\right) e^{-i k_{1} \cdot\left(y-\frac{z_{1}}{2}\right)} e^{i k_{1}^{\prime} \cdot\left(y+\frac{z_{1}}{2}\right)} e^{-\frac{i}{2} k_{2}^{\prime} \cdot z_{2}} e^{-\frac{i}{2} k_{2} \cdot z_{2}}\right] .
\end{aligned}
$$

[1] C. Goebel, F. Halzen, and D. M. Scott, Phys. Rev. D 22, 2789 (1980).

[2] N. Paver and D. Treleani, Nuovo Cimento A 70, 215 (1982).

[3] B. Humpert, Phys. Lett. 131B, 461 (1983).

[4] M. Mekhfi, Phys. Rev. D 32, 2371 (1985).

[5] T. Sjostrand and M. Van Zijl, Phys. Rev. D 36, 2019 (1987).

[6] T. Akesson et al. (Axial Field Spectrometer Collaboration), Z. Phys. C 34, 163 (1987).
[7] B. Blok, Yu. Dokshitzer, L. Frankfurt, and M. Strikman, Eur. Phys. J. C 72, 1963 (2012).

[8] B. Blok, Yu. Dokshitzer, L. Frankfurt, and M. Strikman, Phys. Rev. D 83, 071501 (2011).

[9] B. Blok and M. Strikman, Adv. Ser. Dir. High Energy Phys. 29, 63 (2018).

[10] G. Aad et al. (ATLAS Collaboration), New J. Phys. 15, 033038 (2013). 
[11] M. Diehl, J. R. Gaunt, D. Ostermeier, P. Pll, and A. Schäfer, J. High Energy Phys. 01 (2016) 076.

[12] A. V. Manohar and W. J. Waalewijn, Phys. Rev. D 85, 114009 (2012).

[13] M. Diehl and A. Schafer, Phys. Lett. B 698, 389 (2011).

[14] M. Diehl, D. Ostermeier, and A. Schafer, J. High Energy Phys. 03 (2012) 089.

[15] G. Calucci and D. Treleani, Phys. Rev. D 60, 054023 (1999).

[16] For reviews on generalized parton distributions and DVCS, see M. Diehl, Phys. Rep. 388, 41 (2003); A. V. Belitsky and A. V. Radyushkin, Phys. Rep. 418, 1 (2005); K. Goeke, M. V. Polyakov, and M. Vanderhaeghen, Prog. Part. Nucl. Phys. 47, 401 (2001).

[17] P. J. Mulders and R. D. Tangerman, Nucl. Phys. B461, 197 (1996); V. Barone, A. Drago, and P. G. Ratcliffe, Phys. Rep. 359, 1 (2002); A. Bacchetta, M. Diehl, K. Goeke, A. Metz, P. J. Mulders, and M. Schlegel, J. High Energy Phys. 02 (2007) 093.

[18] M. Rinaldi and F. A. Ceccopieri, Phys. Rev. D 97, 071501 (2018).

[19] M. Aaboud et al. (ATLAS Collaboration), J. High Energy Phys. 11 (2016) 110.

[20] R. Aaij et al. (LHCb Collaboration), J. High Energy Phys. 06 (2017) 047; 10 (2017) 068(E).

[21] S. Chatrchyan et al. (CMS Collaboration), J. High Energy Phys. 03 (2014) 032.

[22] A. M. Sirunyan et al. (CMS Collaboration), J. High Energy Phys. 02 (2018) 032.

[23] M. Aaboudet al. (ATLAS Collaboration), Eur. Phys. J. C 77, 76 (2017).

[24] J. P. Lansberg and H. S. Shao, Phys. Lett. B 751, 479 (2015).

[25] H. M. Chang, A. V. Manohar, and W. J. Waalewijn, Phys. Rev. D 87, 034009 (2013).

[26] M. Rinaldi, S. Scopetta, and V. Vento, Phys. Rev. D 87, 114021 (2013).

[27] M. Rinaldi, S. Scopetta, M. Traini, and V. Vento, J. High Energy Phys. 12 (2014) 028.

[28] M. Rinaldi and F. A. Ceccopieri, Phys. Rev. D 95, 034040 (2017).

[29] W. Broniowski and E. Ruiz Arriola, Few-Body Syst. 55, 381 (2014).

[30] W. Broniowski, E. Ruiz Arriola, and K. Golec-Biernat, FewBody Syst. 57, 405 (2016).

[31] M. Rinaldi, S. Scopetta, M. Traini, and V. Vento, Eur. Phys. J. C 78, 781 (2018).

[32] T. Kasemets and A. Mukherjee, Phys. Rev. D 94, 074029 (2016).

[33] S. Cotogno, T. Kasemets, and M. Myska, Phys. Rev. D 100, 011503 (2019).
[34] M. G. Echevarria, T. Kasemets, P. J. Mulders, and C. Pisano, J. High Energy Phys. 04 (2015) 034.

[35] M. Diehl and T. Kasemets, J. High Energy Phys. 05 (2013) 150.

[36] T. Kasemets and M. Diehl, J. High Energy Phys. 01 (2013) 121.

[37] M. Rinaldi and F. A. Ceccopieri, J. High Energy Phys. 09 (2019) 097.

[38] M. Rinaldi, S. Scopetta, M. Traini, and V. Vento, Phys. Lett. B 752, 40 (2016).

[39] M. Diehl, J. R. Gaunt, P. Ploessl, and A. Schafer, SciPost Phys. 7, 017 (2019).

[40] M. Diehl, P. Pll, and A. Schfer, Eur. Phys. J. C 79, 253 (2019).

[41] B. Blok, Yu. Dokshitzer, L. Frankfurt, and M. Strikman, Eur. Phys. J. C 74, 2926 (2014).

[42] G. S. Bali, P. C. Bruns, L. Castagnini, M. Diehl, J. R. Gaunt, B. Gläßle, A. Schäfer, A. Sternbeck, and C. Zimmermann, J. High Energy Phys. 12 (2018) 061.

[43] M. Rinaldi, S. Scopetta, M.Traini, and V. Vento, J. High Energy Phys. 10 (2016) 063.

[44] M. Diehl, T. Kasemets, and S. Keane, J. High Energy Phys. 05 (2014) 118.

[45] J. Gaunt and W.-J. Stirling, J. High Energy Phys. 03 (2010) 005 .

[46] A. M. Snigirev, Phys. Rev. D 83, 034028 (2011).

[47] A. Mukherjee, Phys. Lett. B 517, 109 (2001).

[48] S. J. Brodsky, D. Chakrabarti, A. Harindranath, A. Mukherjee, and J. P. Vary, Phys. Rev. D 75, 014003 (2007); Phys. Lett. B 641, 440 (2006).

[49] W.-M. Zhang and Harindranath, Phys. Rev. D 48, 4881 (1993).

[50] A. Harindranath, R. Kundu, and W.-M. Zhang, Phys. Rev. D 59, 094013 (1999).

[51] B. D. Jones, Ph. D. Thesis, Ohio State University, 1997.

[52] D. Chakrabarti, X. Zhao, H. Honkanen, R. Manohar, P. Maris, and J. P. Vary, Phys. Rev. D 89, 116004 (2014).

[53] V. L. Korotkikh and A. M. Snigirev, Phys. Lett. B 594, 171 (2004).

[54] C. Goebel, F. Halzen, and D. M. Scott, Phys. Rev. D 22, 2789 (1980).

[55] B. Humpert and R. Odorico, Phys. Lett. 154B, 211 (1985).

[56] F. Halzen, P. Hoyer, and W. J. Stirling, Phys. Lett. B 188, 375 (1987).

[57] R. Kirschner, Phys. Lett. 84B, 266 (1979).

[58] V. P. Shelest, A. M. Snigirev, and G. M. Zinovev, Phys. Lett. 113B, 325 (1982).

[59] F. A. Ceccopieri, Phys. Lett. B 697, 482 (2011). 